\title{
ELECTROMAGNETIC LATTICE "INVISIBILITY" OF THE PHOTON CRYSTAL MADE OF MAGNETODIELECTRIC SPHERES IN THE FORM OF OCTAHEDRON
}

\author{
Anatoliy I. KOZAR ${ }^{1,2, *}$ \\ ${ }^{1}$ Department of Physics, Kharkiv National University of Radio Electronics, Kharkiv, Ukraine \\ ${ }^{2}$ Facultyof Automatics and Computerızed Technologies, Kharkiv National University of Radio Electronics, Kharkiv, Ukraine
}

\begin{abstract}
Solution and analysis of a problem on electromagnetic lattice "invisibility" of the photon crystal made of magnetodielectric spheres in the form of octahedron with a cubic crystal lattice are presented.
\end{abstract}

Keywords: Electromagnetic lattice, Photon crystal, Polyimide, Magnetodielectric spheres, Octahedron

\section{INTRODUCTION}

The problem of modeling the phenomenon of "invisibility" of physical bodies in the optical and x-ray wavelength ranges is a serious area of research in applied electrodynamics. The proposed message analyzes the properties of a limited metacrystal in the form of an octahedron with a cubic lattice when a structural (lattice) resonance is excited in it, in which the phenomenon of electromagnetic lattice "invisibility" occurs:

Here we consider the case equivalent to the x-ray optics of crystals, when $a / \lambda^{\prime}=1 ; a / \lambda_{g}: 1, d, h, / / \lambda^{\prime}: 1 ;$ where $a$ is the radius of the spheres; $\lambda^{\prime}, \lambda_{g}$ - the scattered wavelengths outside and inside the spheres, $d_{1} h_{1} /$ - lattice constants. The solution to the problem is obtained on the basis of the integral equations of Fredholm electrodynamics of the 2nd kind [1, 2, 3, 4].

The numerical results presented in the message are valid not only for macro- but also for micro- and nanocases, if the geometric characteristics of the data are multiplied by conversion factors, by $10^{-4}$ or $10^{-7}$, respectively

\section{MAIN PART}

The scattered field from the known internal field of the scatterers is determined through the electric $\stackrel{\mathbf{u}_{e}}{P}$ and magnetic $\stackrel{\mathbf{u}_{m}}{P}$ potentials of the Hertz spatial lattice $[1,2,3]$

$$
\begin{aligned}
& {\stackrel{\mathrm{r}}{E_{s c a t}}}^{\stackrel{\mathrm{r}}{\mathrm{r}}}=\left(\nabla \nabla+k^{2} \varepsilon_{0} \mu_{0}\right) \stackrel{\mathrm{u}_{e}}{P}-i k \mu_{0}\left[\nabla, \stackrel{\mathbf{u}_{m}}{P}\right], \\
& {\stackrel{H}{H_{s c a t}}}=\left(\nabla \nabla+k^{2} \varepsilon_{0} \mu_{0}\right) \stackrel{\mathbf{u}_{m}}{P}+i k \varepsilon_{0}\left[\nabla, \stackrel{\mathbf{u}_{e}}{P}\right] .
\end{aligned}
$$

The Hertz potential $\stackrel{\mathbf{u}_{e}}{P}$ of the field scattered by the system of lattices over the known internal field of individual scatterers is represented as a superposition of the Hertz potentials of the individual spheres of the lattices

\footnotetext{
*Corresponding Author: anatoliy.kozar@nure.ua
}

Received: 11.10.2019 Published: 16.12.2019 


$$
\begin{aligned}
& {\stackrel{\mathbf{u}_{e}}{P}}^{\mathrm{r}}(\stackrel{\mathrm{r}}{r}, t)=\sum_{c=1}^{c}\left[\sum_{p} \sum_{s} \sum_{t} \frac{1}{k_{1}^{3}}\left(\sin k_{1} a_{c}-k_{1} a_{c} \cos k_{1} a_{c}\right) \mathrm{x}\right. \\
& \left.\times\left(\frac{\varepsilon_{c \rho \phi}}{\varepsilon_{0}}-1\right) \underset{E_{c(p, s, t)}^{\mathrm{r}}}{\mathrm{r}}\left(\stackrel{r}{r}^{\prime}, t\right) \frac{e^{-i k_{1} r_{c(p, s, t)}}}{r_{c(p, s, t)}}\right]_{c}, \\
& r_{c(p, s, t)}=\sqrt{\left(x-x_{c, s}\right)^{2}+\left(y-y_{c, t}\right)^{2}+\left(z-z_{c, p}\right)^{2}} \text {, }
\end{aligned}
$$

where the coordinates $(x, y, z)$ determine the field observation points outside the spheres, and $\left(x_{c, s}, y_{c, t}, z_{c, p}\right)$

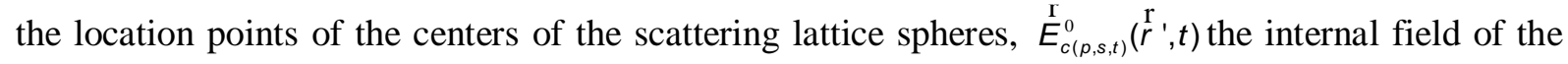
spheres, which is found from the algebraic system of inhomogeneous equations of the quasistationary approximation $[1,3]$.

From (1), taking into account (2), the field scattered by the system of orthogonal lattices is found in the form

$$
\begin{aligned}
& \stackrel{\mathrm{r}}{E_{s c a t}}(\stackrel{\mathrm{r}}{r}, t)=\sum_{c=1}^{c}\left[\sum_{p} \sum_{s} \sum_{t} \frac{1}{k_{1}^{3}}\left(\sin k_{1} a_{c}-k_{1} a_{c} \cos k_{1} a_{c}\right) \times\right.
\end{aligned}
$$

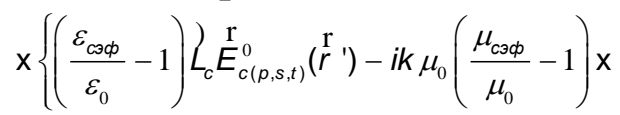

$$
\begin{aligned}
& \left.\left.\left.x \dot{P}_{c}{\stackrel{r}{H_{c(p, s, t)}^{0}}}_{\left(r^{\prime}\right)}^{\mathrm{r}}\right)\right\} e^{i\left(\omega t-k_{1} r_{c(p, s, t)}\right)}\right]_{c},
\end{aligned}
$$

where $\dot{L}_{c}$ and $\dot{P}_{c}$ are the functional scattering matrices. Expression (4) describes the propagating and decaying components of the scattered field inside and outside the crystal in the Fresnel and Fraunhofer zones.

The total field at an arbitrary point in the medium that is outside the spheres is defined as

$$
\begin{aligned}
& \stackrel{\mathrm{r}}{E}(\stackrel{\mathrm{r}}{r}, t)=\stackrel{\stackrel{\mathrm{r}}{E}}{\mathrm{r}}(\stackrel{\mathrm{r}}{r}, t)+\stackrel{\mathrm{I}}{E}_{s c a t}(\stackrel{\mathrm{r}}{r}, t), \\
& \stackrel{\mathrm{r}}{H}(\stackrel{\mathrm{r}}{r}, t)=\stackrel{\mathrm{r}}{H}_{0}(\stackrel{\mathrm{r}}{r}, t)+\stackrel{\mathrm{r}}{H}_{\text {scat }}(\stackrel{\mathrm{r}}{r}, t)
\end{aligned}
$$

where $\stackrel{\mathrm{I}}{E}_{0}(\stackrel{\mathrm{r}}{r}, t), \stackrel{\mathrm{r}}{H}_{0}(\stackrel{\mathrm{r}}{r}, t)$ are the unperturbed fields of the scattered wave.

The energy density of the scattered (1) and total (2) electromagnetic fields can be found using the relation

$$
\omega=\frac{1}{8 \pi}\left(\stackrel{\mathrm{r}}{E^{2}}+\stackrel{\mathrm{r}}{H^{2}}\right)
$$

Expressions (1) and (6) are numerically analyzed for a resonant crystal in the form of an octahedron, the results of which are shown in Figure 1, 2. The relationship between the resonant length $\lambda_{p}^{c m}$ of the scattered plane wave and the cubic lattice constant $d$ of the crystal is selected in the form [3]

$$
\lambda_{p}^{c m}=0,8 d
$$




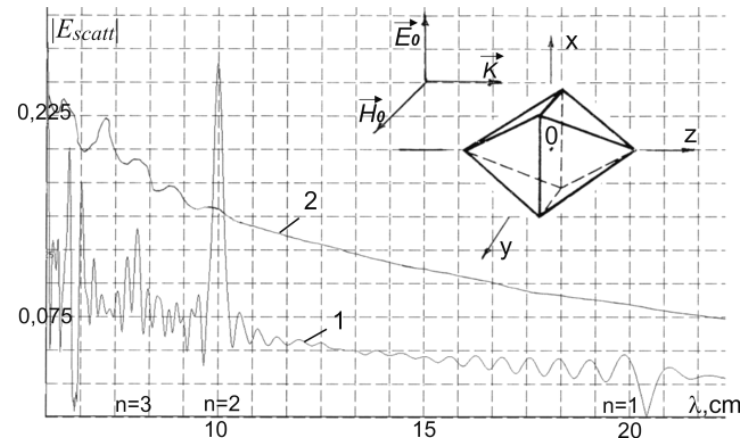

a)

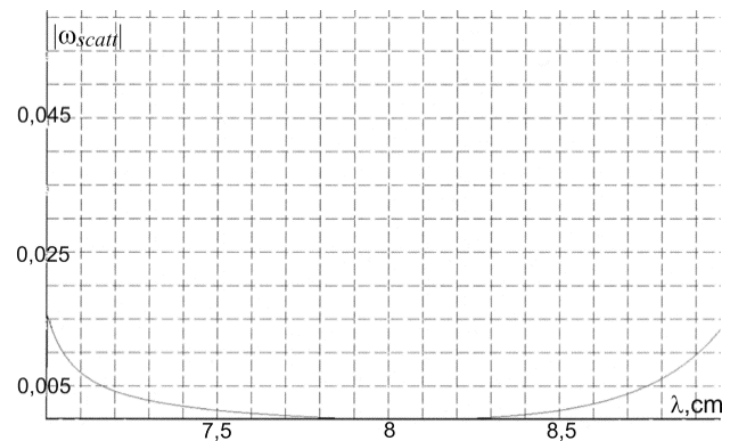

b)

Figure 1. Dispersion dependences for $\left|E_{\text {scat }}\right|$ and $\left|\omega_{\text {scat }}\right|$ octahedron

Under this condition, a crystal (lattice) resonance arises in the crystal with the index $n=3$ (Fig. 1a) and the associated phenomenon of electromagnetic lattice "invisibility", when the scattered wave does not experience reflection, but mainly passes through the crystal (Fig. 2 a, b) Here, the shape of the resonance curves is determined by the algebraic sum of the fields with the corresponding phase factors (4).

This effect is associated with the appearance of zones of resonant transmission of the scattered wave (Figure $1 \mathrm{~b}$ ).

The position of dependences $\left|\omega_{\text {scat }}\right|$ (6) at a fixed distance on both sides of the crystal along the $\mathrm{z}$ axis in the Fresnel and Fraunhofer zones in the direction of propagation of the scattered wave allows us to estimate the width of the zones of resonant wave propagation.

Here in the crystal the number of spheres is $\mathrm{N}=64000$; radius of spheres $-a=0.5 \mathrm{~cm}$; permeability of spheres $-\varepsilon=95, \mu=1$ and the environment $-\varepsilon_{0}=\mu_{0}=1$; cubic crystal lattice constant $-\mathrm{d}=\mathrm{h}=1=10 \mathrm{~cm}$.

Figure 1a shows the dispersion dependences $\left|E_{\text {scat }}\right|$ (4) in the center of the octahedron (curve 1) and in the vicinity of its vertex (curve 2). The indices $n=1,2,3 \ldots$ determine the number of structural resonance [3].

Figurelb shows the dispersion dependences (6) outside the octahedron for distances along the $\mathrm{z}$ axis $=$ $10000 \mathrm{~cm}$.

In Figure 2a and $2 b$, we consider the structure of the internal field of the resonant octahedron in the direction of the $\mathrm{z}$ axis for $\left|E_{\text {scat }}\right|$ and $\left|\omega_{\text {scat }}\right|$ (6).

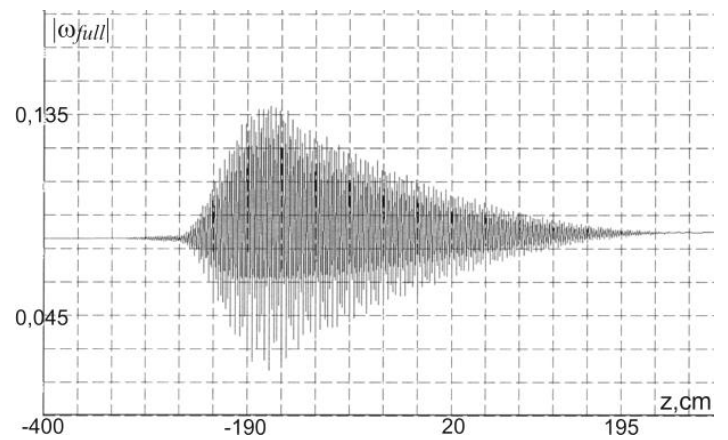

a)

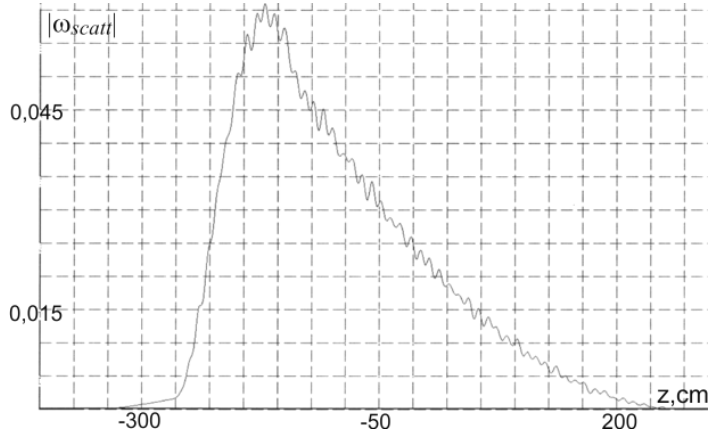

b)

Figure 2. Dependences for $\left|E_{\text {scat }}\right|$ and $\left|\omega_{\text {scat }}\right|$ the internal field of the octahedron 


\section{CONCLUSION}

Using structural (lattice) resonances of crystals, the occurrence of which is associated with the presence of a certain shape of the external surface envelope of the crystal, it is possible to create conditions for the appearance of resonant lattice "invisibility" during the scattering of electromagnetic waves by crystals and form the structure of the internal field of the crystal.

The results of the study presented in this report can be used to create devices that use "stealth" technologies and to analyze resonant lattice phenomena in x-ray optics of real crystals.

\section{REFERENCES}

[1] Kozar AI. Electromagnetic Wave Scattering with Special Spatial Lattices of Magnetodielectric Spheres // J. Telecommunication and Radio Engineering. - New York, N.Y. (USA): Begell House Inc. 2004; Vol. 61, No.9. - p.p. 734-749.

[2] Kozar AI. Resonant Degenerate Crystal Made of Spheres Located Magnetodielectric Medium, International Journal of Electromagnetics and Applications, Vol. 3, No.2, 2013, pp. 15-19. doi: 10.5923/ j.idea.20130302.02.

[3] Kozar AI. Resonant metacrystals of small magnetodielectric spheres: monograph / A.I. Kozar, Ukraine - Kharkiv: KNURE, 2014. - 352 p. (in Russian).

[4] Khyzhnyak NA. The Green function of Maxwell's equations for inhomogeneous media. J. Technical Physics. 1958. Vol. 28, № 7, pp. 1952-1610 (in Russian). 